Math. Model. Nat. Phenom.

Vol. 2, No. 2, 2007, pp. 20-39

\title{
Convective Instability of Reaction Fronts in Porous Media
}

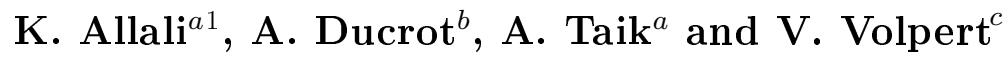 \\ ${ }^{a}$ Department of Mathematics, University Hassan II, Mohammedia, Morocco \\ ${ }^{b}$ University Bordeaux 2, UMR 5466 CNRS, 33076 Bordeaux, France \\ ${ }^{c}$ University Lyon 1, UMR 5208 CNRS, 69622 Villeurbanne, France
}

\begin{abstract}
We study the influence of natural convection on stability of reaction fronts in porous media. The model consists of the heat equation, of the equation for the depth of conversion and of the equations of motion under the Darcy law. Linear stability analysis of the problem is fulfilled, the stability boundary is found. Direct numerical simulations are performed and compared with the linear stability analysis.
\end{abstract}

Key words: natural convection, Darcy law, Boussinesq approximation, linear stability analysis

AMS subject classification: $35 \mathrm{~K} 57,76 \mathrm{~S} 05,76 \mathrm{E} 15$

\section{Introduction}

Propagation of reaction fronts can be accompanied by various instabilities. Under the constant density approximation with a single stage exothermic chemical reaction, front propagation is described by the reaction-diffusion system of equations (2.1), (2.2). It can exhibit the thermo-diffusional instability that can manifest itself as one-dimensional pulsations, various multi-dimensional periodic in time waves, cellular patterns and so on (see $[15,16]$ and the references therein). The stability conditions are determined by the Lewis number $L=\kappa / d$ and by the Zeldovich number $Z$ defined below.

If the density of the medium depends on temperature, then its expansion in the reaction zone can result in the hydrodynamic instability first studied in [8,9] (see [11] for recent results and the literature review).

\footnotetext{
${ }^{1}$ Corresponding author. Email: allali@fstm.ac.ma
} 
Propagation of reaction fronts in fluids under the gravity field can result in the convective instability. If the front propagates upwards and the reaction is exothermic, then it heats cold original reactants from below. If the frontal Rayleigh number [7] is sufficiently large, then the convective instability appears. For downward propagating fronts, though the reaction zone heats cold reactants from above, the convective instability can also occur [6] unlike in a layer of a liquid heated from above. This problem has been studied studied by a formal asymptotic analysis in the limit of large Zeldovich numbers.

Propagation of reaction fronts in a porous medium can be influenced by the interaction of reacting gases or liquids with a porous matrix resulting in fingering $[4,5]$, various phenomena specific for filtration combustion $[1,2,14]$ or for transition from combustion to detonation [3].

In this work we continue the investigation of exothermic reaction fronts in a porous medium. We will study the convective instability and the influence of natural convection on the thermal instability. The contents of the paper are as follow. We introduce the model in Section 2 and reduce it to a singular perturbation problem in Sections 3 and 4. In Section 5 we consider the linearized problem and derive the dispersion relation. The stability conditions given by the linear stability analysis are discussed in Section 6. In Section 7 we present the numerical study of the complete model. Finally in the last section we compare and discuss the analytical and numerical results.

\section{Governing Equations}

To study propagation of exothermic reaction fronts in a porous medium we consider the reaction-diffusion system of equations coupled with the equations of motion corresponding to the Darcy law and the Boussinesq approximation. We consider the upward propagation of the reaction front, that is in the direction opposite to the gravity. The process is described by the following model:

$$
\begin{gathered}
\frac{\partial T}{\partial t}+\mathbf{v} \cdot \nabla T=\kappa \Delta T+q K(T) \phi(\alpha), \\
\frac{\partial \alpha}{\partial t}+\mathbf{v} \cdot \nabla \alpha=d \Delta \alpha+K(T) \phi(\alpha), \\
\mathbf{v}+\frac{K_{p}}{\mu} \nabla p=\frac{g \beta K_{p}}{\mu} \rho\left(T-T_{0}\right) \gamma, \\
\nabla \cdot \mathbf{v}=0 .
\end{gathered}
$$

This system is considered in the whole space $\mathbb{R}^{2}$. It is supplemented by the following conditions

$$
\begin{aligned}
& T=T_{i}, \quad \alpha=0 \text { and } \mathbf{v}=0 \text { when } y \rightarrow+\infty \\
& T=T_{b}, \quad \alpha=1 \text { and } \mathbf{v}=0 \text { when } y \rightarrow-\infty
\end{aligned}
$$


Here $\beta$ denotes the coefficient of thermal expansion, $\mu$ the viscosity, $K_{p}$ the permeability of the porous media and $\gamma$ is the unit vector in the vertical direction (upward), $T$ is the temperature, $\alpha$ the depth of conversion, $\mathbf{v}=\left(v_{x}, v_{y}\right)$ the velocity, $p$ the pressure, $\kappa$ the coefficient of thermal diffusivity, $d$ the diffusion coefficient, $q$ the adiabatic heat release, $g$ the gravity acceleration, $\rho$ is the density. Finally, $\nabla$ and $\Delta$ denote the standard differential operators

$$
\nabla=\left(\frac{\partial}{\partial x}, \frac{\partial}{\partial y}\right), \Delta=\frac{\partial^{2}}{\partial x^{2}}+\frac{\partial^{2}}{\partial y^{2}}
$$

In addition $T_{0}$ is the mean value of the temperature, $T_{i}$ is an initial temperature while $T_{b}$ is the temperature of the reacted mixture given by $T_{b}=T_{i}+q$. The function $K(T) \phi(\alpha)$ describes the reaction rate where the temperature dependence is given by the Arrhenius exponential:

$$
K(T)=k_{0} \exp \left(-\frac{E}{R_{0} T}\right)
$$

$E$ is the activation energy, $R_{0}$ the universal gas constant and $k_{0}$ the pre-exponential factor. The asymptotic analysis of this problem will be carried out in the limit of large activation energies specific for combustion and polymerization fronts.

For the asymptotic analysis of the problem we will consider the zero order reaction,

$$
\phi(\alpha)=\left\{\begin{array}{l}
1 \text { if } \alpha<1 \\
0 \text { if } \alpha=1
\end{array}\right.
$$

while for the numerical simulations it is more convenient to use the first order reaction, $\phi(\alpha)=1-\alpha$. It is known that the qualitative behavior of reaction fronts for the zero and for the first order reactions is the same.

Problem (2.1)-(2.5) includes the heat equation, chemical reaction and the motion of an incompressible liquid in a porous medium. The heat release due to the exothermic chemical reaction results in a nonhomogeneous temperature distribution and can lead to the convective instability.

If the medium is at rest, that is $\mathbf{v}=0$, then there exists a stationary propagating front with the velocity and temperature distribution that can be found asymptotically for large values of the Zeldovich number $Z=q E / R_{0} T_{b}^{2}$. In the case of the zero order reaction we have

$$
\begin{gathered}
c^{2}=\frac{2 k_{0} \kappa}{q} \frac{R_{0} T_{b}^{2}}{E} \exp \left(\frac{-E}{R_{0} T_{b}}\right), \\
T(\bar{y}, t)= \begin{cases}T_{b} & \text { if } \bar{y}<0, \\
T_{i}+\left(T_{b}-T_{i}\right) \exp \left(\frac{-\bar{y} c}{\kappa}\right) & \text { if } \bar{y}>0,\end{cases} \\
\alpha(\bar{y}, t)= \begin{cases}1 & \text { if } \bar{y}<0, \\
0 & \text { if } \bar{y}>0 .\end{cases}
\end{gathered}
$$


We now introduce the dimensionless spatial variable $x^{\prime}=x c_{1} / \kappa, y^{\prime}=y c_{1} / \kappa$, time $t^{\prime}=$ $t c_{1}^{2} / \kappa d$, velocity $\mathbf{v} / c_{1}$ and pressure $p \kappa \mu / K_{p}$ with $c_{1}=c / \sqrt{2}$. Denoting $\theta=\left(T-T_{b}\right) / q$ and keeping for convenience the same notation for the other variables, we re-write system (2.1)-(2.4) in the following form

$$
\begin{gathered}
\frac{\partial \theta}{\partial t}+\mathbf{v} \cdot \nabla \theta=\Delta \theta+W_{Z}(\theta) \phi(\alpha) \\
\frac{\partial \alpha}{\partial t}+\mathbf{v} \cdot \nabla \alpha=\Lambda \Delta \alpha+W_{Z}(\theta) \phi(\alpha) \\
\mathbf{v}+\nabla p=R_{p}\left(\theta+\theta_{0}\right) \gamma \\
\nabla \cdot \mathbf{v}=0
\end{gathered}
$$

with

$$
W_{Z}(\theta)=Z \exp \left(\frac{\theta}{Z^{-1}+\delta \theta}\right)
$$

and the function $\phi$ defined in (2.8). Here $\Lambda=d / \kappa$ is the inverse of Lewis number, $R_{p}=$ $K_{p} c_{1}^{2} P^{2} R / \mu^{2}$, where $R$ is the Rayleigh number and $P$ is the Prandtl number defined by $R=g \beta q \kappa^{2} / \mu c_{1}^{3}$ and $P=\mu / \kappa$, respectively. In addition, the parameters $\delta$ and $\theta_{0}$ are defined by $\delta=R_{0} T_{b} / E$ and $\theta_{0}=\left(T_{b}-T_{0}\right) / q$. Finally boundary conditions (2.5) re-write:

$$
\begin{gathered}
y \rightarrow+\infty, \quad \theta=-1, \quad \alpha=0 \text { and } \mathbf{v}=0 \\
y \rightarrow-\infty, \quad \theta=0, \quad \alpha=1 \text { and } \mathbf{v}=0
\end{gathered}
$$

The linear stability analysis is carried out for the case $\Lambda=0$ which corresponds to a liquid mixture. For the direct numerical simulations we consider small positive values of $\Lambda$.

\section{Approximation of infinitely narrow reaction zone}

To study this problem analytically we reduce it to a singular perturbation problem where the reaction zone is replaced by a reaction surface and the reaction term is neglected outside the reaction zone (Zeldovich - Frank-Kamanetsky method). This is a conventional approach for combustion problems. We perform a formal asymptotic analysis with $\epsilon=1 / Z$ taken as the small parameter and we obtain a closed interface problem.

Let $y=\zeta(t, x)$ be the location of the reaction zone and define the new independent variable as

$$
y_{1}=y-\zeta(t, x)
$$

We introduce new unknown functions $\theta_{1}, \alpha_{1}, \mathbf{v}_{1}, p_{1}$ :

$$
\begin{aligned}
& \theta(t, x, y)=\theta_{1}\left(t, x, y_{1}\right), \quad \alpha(t, x, y)=\alpha_{1}\left(t, x, y_{1}\right) \\
& \mathbf{v}(t, x, y)=\mathbf{v}_{1}\left(t, x, y_{1}\right), \quad p(t, x, y)=p_{1}\left(t, x, y_{1}\right)
\end{aligned}
$$


We re-write the equations in the form (the index 1 for the dependent variables is omitted):

$$
\begin{gathered}
\frac{\partial \theta}{\partial t}-\frac{\partial \theta}{\partial y_{1}} \frac{\partial \zeta}{\partial t}+\mathbf{v} \cdot \widetilde{\nabla} \theta=\widetilde{\Delta} \theta+W_{Z}(\theta) \phi(\alpha) \\
\frac{\partial \alpha}{\partial t}-\frac{\partial \alpha}{\partial y_{1}} \frac{\partial \zeta}{\partial t}+\mathbf{v} \cdot \widetilde{\nabla} \alpha=W_{Z}(\theta) \phi(\alpha) \\
\mathbf{v}+\widetilde{\nabla} p=R_{p}\left(\theta+\theta_{0}\right) \gamma \\
\frac{\partial v_{x}}{\partial x}-\frac{\partial v_{x}}{\partial y_{1}} \frac{\partial \zeta}{\partial x}+\frac{\partial v_{y}}{\partial y_{1}}=0
\end{gathered}
$$

where we have set

$$
\begin{gathered}
\widetilde{\Delta}=\frac{\partial^{2}}{\partial x^{2}}+\frac{\partial^{2}}{\partial y_{1}^{2}}-2 \frac{\partial \zeta}{\partial x} \frac{\partial^{2}}{\partial x \partial y_{1}}+\left(\frac{\partial \zeta}{\partial x}\right)^{2} \frac{\partial^{2}}{\partial y_{1}^{2}}-\frac{\partial^{2} \zeta}{\partial x^{2}} \frac{\partial}{\partial y_{1}} \\
\widetilde{\nabla}=\left(\frac{\partial}{\partial x}-\frac{\partial \zeta}{\partial x} \frac{\partial}{\partial y_{1}}, \frac{\partial}{\partial y_{1}}\right) .
\end{gathered}
$$

We use matched asymptotic expansions. We look for the outer solution of the problem in the form of the expansion

$$
\begin{aligned}
& \theta=\theta^{0}+\epsilon \theta^{1}+\ldots, \quad \alpha=\alpha^{0}+\epsilon \alpha^{1}+\ldots \\
& \mathbf{v}=\mathbf{v}^{0}+\epsilon \mathbf{v}^{1}+\ldots, \quad p=p^{0}+\epsilon p^{1}+\ldots
\end{aligned}
$$

Here $\left(\theta^{0}, \alpha^{0}, \mathbf{v}^{0}\right)$ is the dimensionless form of the basic solution given in (2.9)-(2.11).

In order to obtain the matching conditions across the reaction zone we consider the inner problem and we introduce the stretched coordinate $\eta=y_{1} / \epsilon$. We look for the inner solution in the form of the expansion

$$
\begin{aligned}
& \theta=\epsilon \tilde{\theta}^{1}+\ldots, \quad \alpha=\tilde{\alpha}^{0}+\epsilon \tilde{\alpha}^{1}+\ldots \\
& \mathbf{v}=\tilde{\mathbf{v}}^{0}+\epsilon \tilde{\mathbf{v}}^{1}+\ldots, \quad p=\tilde{p}^{0}+\epsilon \tilde{p}^{1}+\ldots, \quad \zeta=\tilde{\zeta}^{0}+\epsilon \tilde{\zeta}^{1}+\ldots
\end{aligned}
$$

Substituting these expansions into (3.21)-(3.24), we obtain: order $\epsilon^{-1}$

$$
\begin{gathered}
\left(1+\left(\frac{\partial \tilde{\zeta}^{0}}{\partial x}\right)^{2}\right) \frac{\partial^{2} \tilde{\theta}^{1}}{\partial \eta^{2}}+\exp \left(\frac{\tilde{\theta}^{1}}{1+\delta \tilde{\theta}^{1}}\right) \phi\left(\tilde{\alpha}^{0}\right)=0 \\
-\frac{\partial \tilde{\alpha}^{0}}{\partial \eta} \frac{\partial \tilde{\zeta}^{0}}{\partial t}-\frac{\partial \tilde{\alpha}^{0}}{\partial \eta}\left(\tilde{v}_{x}^{0} \frac{\partial \tilde{\zeta}^{0}}{\partial x}-\tilde{v}_{y}^{0}\right)=\exp \left(\frac{\tilde{\theta}^{1}}{1+\delta \tilde{\theta}^{1}}\right) \phi\left(\tilde{\alpha}^{0}\right), \\
\frac{\partial \tilde{p}^{0}}{\partial \eta}=0 \\
\tilde{v}_{x}^{0}+\frac{\partial \tilde{p}^{0}}{\partial x}-\frac{\partial \tilde{\zeta}^{0}}{\partial x} \frac{\partial \tilde{p}^{1}}{\partial \eta}=0
\end{gathered}
$$




$$
-\frac{\partial \tilde{v}_{x}^{0}}{\partial \eta} \frac{\partial \tilde{\zeta}^{0}}{\partial x}+\frac{\partial \tilde{v}_{y}^{0}}{\partial \eta}=0
$$

order $\epsilon^{0}$

$$
\tilde{v}_{y}^{0}+\frac{\partial \tilde{p}^{1}}{\partial \eta}=-R_{p} \theta_{0}
$$

Let us consider both the outer and the inner expansion of the temperature (we use the same technique for the variables $\alpha$ and $\mathbf{v}$ ) and recall that $\eta=y_{1} / \epsilon$ :

$$
\begin{gathered}
\theta\left(x, y_{1}\right)=\theta^{0}\left(x, y_{1}\right)+\epsilon \theta^{1}\left(x, y_{1}\right)+\epsilon^{2} \theta^{2}\left(x, y_{1}\right)+\ldots, \\
\theta(x, \epsilon \eta)=\epsilon \tilde{\theta}^{1}(x, \eta)+\epsilon^{2} \tilde{\theta}^{2}(x, \eta)+\ldots
\end{gathered}
$$

We write the outer solution in terms of the inner variable $\eta$ and we use the Taylor expansion:

$$
\begin{aligned}
\theta\left(x, y_{1}\right) & =\quad \theta^{0}(x, 0)+\epsilon\left(\frac{\partial \theta^{0}}{\partial \eta}(x, 0) \eta+\theta^{1}(x, 0)\right) \\
& +\epsilon^{2}\left(\frac{1}{2} \frac{\partial^{2} \theta^{0}}{\partial \eta^{2}}(x, 0) \eta^{2}+\frac{\partial \theta^{1}}{\partial \eta}(x, 0) \eta+\theta^{2}(x, 0)\right)+\ldots
\end{aligned}
$$

The zero order terms with respect to $\epsilon$ correspond to the stationary solution. Equating the first order terms and taking into account that $\frac{\partial \theta^{0}}{\partial \eta}(x, 0-)=0$, we obtain, using the matching principle [12], [13], the following matching conditions:

$$
\begin{gathered}
\eta \rightarrow+\infty:\left.\quad \tilde{\theta}^{1} \sim \theta^{1}\right|_{y_{1}=0+}+\left.\eta \frac{\partial \theta^{0}}{\partial y_{1}}\right|_{y_{1}=0+}, \quad \tilde{\alpha}^{0} \rightarrow 0,\left.\quad \tilde{\mathbf{v}}^{0} \rightarrow \mathbf{v}^{0}\right|_{y_{1}=0+}, \\
\eta \rightarrow-\infty:\left.\quad \tilde{\theta}^{1} \rightarrow \theta^{1}\right|_{y_{1}=0-}, \quad \tilde{\alpha}^{0} \rightarrow 1,\left.\quad \tilde{\mathbf{v}}^{0} \rightarrow \mathbf{v}^{0}\right|_{y_{1}=0-} .
\end{gathered}
$$

From (3.31) we obtain that $\tilde{p}^{0}$ does not depend on $\eta$, which implies that at the leading order the pressure is continuous through the interface. Next, denoting by $s$ the quantity

$$
s=\tilde{v}_{x}^{0} \frac{\partial \tilde{\zeta}^{0}}{\partial x}-\tilde{v}_{y}^{0}
$$

we obtain from (3.33) that $s$ does not depend on $\eta$. Finally from (3.32), (3.34) and (3.37) we easily obtain that $\tilde{v}_{x}^{0}$ and $\tilde{v}_{y}^{0}$ do not depend on $\eta$, which provides the continuity of the velocity across the interface.

We next derive the jump conditions for the temperature from (3.29), in the same way as it is usually done for combustion problems. From (3.30) it follows that $\tilde{\alpha}^{0}$ is a monotone function of $\eta$ and $0<\tilde{\alpha}^{0}<1$. Since we consider zero-order reaction, we have $\phi\left(\tilde{\alpha}^{0}\right) \equiv 1$. From (3.29) we conclude that $\frac{\partial^{2} \tilde{\theta}^{1}}{\partial \eta^{2}} \leq 0$. From (3.36) we have $\frac{\partial \tilde{\theta}^{1}}{\partial \eta}=0$ at $\eta=-\infty$. Then $\frac{\partial \tilde{\theta}^{1}}{\partial \eta} \leq 0$ and $\tilde{\theta}^{1}$ is a monotone function. 
We multiply (3.29) by $\frac{\partial \tilde{\theta}^{1}}{\partial \eta}$ and integrate with respect to $\eta$ from $-\infty$ to $+\infty$ taking into account $(3.35),(3.36)\left(\tilde{\theta}^{1}\right.$ changes from $\left.\theta^{1}\right|_{y_{1}=0-}$ to $-\infty$ when $\eta$ changes from $-\infty$ to $\left.+\infty\right)$ :

$$
\left.\left(\frac{\partial \tilde{\theta}^{1}}{\partial \eta}\right)^{2}\right|_{\eta=+\infty}-\left.\left(\frac{\partial \tilde{\theta}^{1}}{\partial \eta}\right)^{2}\right|_{\eta=-\infty}=\frac{2}{A} \int_{-\infty}^{\theta^{1} \mid y_{1}=0-} \exp \left(\frac{\tau}{1+\delta \tau}\right) d \tau
$$

where we have set

$$
A=1+\left(\frac{\partial \tilde{\zeta}^{0}}{\partial x}\right)^{2}
$$

Next, adding (3.29) and (3.30) and integrating we obtain

$$
\left.\frac{\partial \tilde{\theta}^{1}}{\partial \eta}\right|_{\eta=+\infty}-\left.\frac{\partial \tilde{\theta}^{1}}{\partial \eta}\right|_{\eta=-\infty}=-\frac{1}{A}\left(\frac{\partial \tilde{\zeta}^{0}}{\partial t}+s\right) .
$$

Truncating the outer expansion (3.27), we have $\theta \approx \theta^{0}, \quad \zeta^{0} \approx \zeta, \mathbf{v} \approx \mathbf{v}^{0}$. From the inner expansion (3.28) we obtain $Z \theta \approx \tilde{\theta}^{1}$, and from the matching condition (3.36), $\left.\tilde{\theta}^{1}\right|_{\eta=-\infty} \approx$ $\left.\left.\theta^{1}\right|_{y_{1}=0-} \approx Z \theta\right|_{y_{1}=0-}$. Thus,

$$
\theta^{0} \approx \theta,\left.\left.\quad \theta^{1}\right|_{y_{1}=0-} \approx Z \theta\right|_{y_{1}=0}, \quad \zeta^{0} \approx \zeta, \quad \mathbf{v} \approx \mathbf{v}^{0}
$$

We finally obtain the jump conditions (with the change of variables $\tau \rightarrow Z \tau$ under the integral)

$$
\begin{gathered}
\left.\left(\frac{\partial \theta}{\partial y_{1}}\right)^{2}\right|_{y_{1}=0+}-\left.\left(\frac{\partial \theta}{\partial y_{1}}\right)^{2}\right|_{y_{1}=0-}=2 Z\left(1+\left(\frac{\partial \zeta}{\partial x}\right)^{2}\right)^{-1} \int_{-\infty}^{\left.\theta\right|_{y_{1}=0}} \exp \left(\frac{\tau}{Z^{-1}+\delta \tau}\right) d \tau \\
\left.\frac{\partial \theta}{\partial y_{1}}\right|_{y_{1}=0+}-\left.\frac{\partial \theta}{\partial y_{1}}\right|_{y_{1}=0-}=-\left(1+\left(\frac{\partial \zeta}{\partial x}\right)^{2}\right)^{-1}\left(\frac{\partial \zeta}{\partial t}+\left.\left(v_{x} \frac{\partial \zeta}{\partial x}-v_{y}\right)\right|_{y_{1}=0}\right) .
\end{gathered}
$$

\section{Formulation of the interface problem}

Here we present the complete formulation of the free boundary problem derived above. We have for $y>\zeta$ (in the unreacted medium)

$$
\begin{gathered}
\frac{\partial \theta}{\partial t}+\mathbf{v} \cdot \nabla \theta=\Delta \theta, \\
\alpha \equiv 0 \\
\mathbf{v}+\nabla p=R_{p}\left(\theta+\theta_{0}\right) \gamma \\
\nabla \cdot \mathbf{v}=0 .
\end{gathered}
$$


The equations in the reacted medium $(y<\zeta)$ have the form

$$
\begin{gathered}
\frac{\partial \theta}{\partial t}+\mathbf{v} \cdot \nabla \theta=\Delta \theta, \\
\alpha \equiv 1, \\
\mathbf{v}+\nabla p=R_{p}\left(\theta+\theta_{0}\right) \gamma, \\
\nabla \cdot \mathbf{v}=0 .
\end{gathered}
$$

We finally complete this system by the following jump conditions at the interface $y=\zeta$ :

$$
\begin{gathered}
{[\theta]=0,\left[\frac{\partial \theta}{\partial y}\right]=\frac{\frac{\partial \zeta}{\partial t}}{1+\left(\frac{\partial \zeta}{\partial x}\right)^{2}}} \\
{\left[\left(\frac{\partial \theta}{\partial y}\right)^{2}\right]=-\frac{2 Z}{1+\left(\frac{\partial \zeta}{\partial x}\right)^{2}} \int_{-\infty}^{\theta(t, x, \zeta)} \exp \left(\frac{s}{1 / Z+\delta s}\right) d s} \\
{[\mathbf{v}]=0 .}
\end{gathered}
$$

Here [ ] denotes the jump at the interface,

$$
[f]=\left.f\right|_{\zeta-0}-\left.f\right|_{\zeta+0}
$$

The free boundary problem is completed by the conditions at infinity

$$
\begin{gathered}
y \rightarrow+\infty, \quad \theta=-1 \text { and } \mathbf{v}=0 \\
y \rightarrow-\infty, \quad \theta=0 \text { and } \mathbf{v}=0
\end{gathered}
$$

\section{Linear stability analysis and dispersion relation}

\subsection{Linearization}

The interface problem presented in the previous section has a travelling wave solution $(\theta, \alpha, \mathbf{v})$,

$$
\theta(t, x, y)=\theta_{s}(y-u t), \quad \alpha(t, x, y)=\alpha_{s}(y-u t), \quad \mathbf{v}=0, \quad \zeta=u t
$$

(in what follows we do not need the explicit expression for the pressure $p$ ). Here

$$
\theta_{s}\left(y_{1}\right)=\left\{\begin{array}{ll}
0 & \text { if } y_{1}<0 \\
e^{-u y_{1}}-1 & \text { if } y_{1}>0
\end{array},\right.
$$


$y_{1}=y-u t$, the number $u$ stands for the wave speed. It can be found from the jump conditions,

$$
u^{2}=2 Z \int_{-\infty}^{0} \exp \left(\frac{\tau}{Z^{-1}+\delta \tau}\right) d \tau
$$

We now introduce the moving coordinate frame, $y_{1}=y-u t$. In these coordinates the travelling wave is a stationary solution of the problem

$$
\begin{gathered}
\frac{\partial \theta}{\partial t}+u \frac{\partial \theta}{\partial y_{1}}+\mathbf{v} \cdot \nabla \theta=\Delta \theta \\
\mathbf{v}+\nabla p=R_{p}\left(\theta+\theta_{0}\right) \gamma \\
\nabla \cdot \mathbf{v}=0
\end{gathered}
$$

together with the jump conditions (4.52)-(4.54).

We next consider a small perturbation of this stationary solution. For that purpose we consider a perturbation of the reaction front, of the temperature and of the velocity:

$$
\begin{gathered}
\zeta(t, x)=u t+\xi(t, x) \\
\theta(t, x, y)=\theta_{s}(y-u t)+\theta_{j}(z) e^{\omega t+i k x}, j=1,2, \\
v_{y}(t, x, y)=v_{j}(z) e^{\omega t+i k x}
\end{gathered}
$$

where

$$
z=y-\zeta(t, x)=y-u t-\xi(t, x), \quad \xi(t, x)=\epsilon_{1} e^{\omega t+i k x},
$$

$j=1$ corresponds to $z<0$ and $j=2$ to $z>0$.

We linearize system (5.59)-(5.61) about the stationary solution and apply rot rot transformation to eliminate the pressure in the equation (5.5). We obtain the following system, for $z<0$ :

$$
\begin{gathered}
\theta_{1}^{\prime \prime}+u \theta_{1}^{\prime}-\left(\omega+k^{2}\right) \theta_{1}=0 \\
v_{1}^{\prime \prime}-k^{2} v_{1}=-R_{p} k^{2} \theta_{1}
\end{gathered}
$$

for $z>0$ :

$$
\begin{gathered}
\theta_{2}^{\prime \prime}+u \theta_{2}^{\prime}-\left(\omega+k^{2}\right) \theta_{2}+v_{2} u e^{-u z}=0, \\
v_{2}^{\prime \prime}-k^{2} v_{2}=-R_{p} k^{2} \theta_{2} .
\end{gathered}
$$

This system is completed with the linearized jump condition (4.52)-(4.54):

$$
\begin{gathered}
\theta_{2}(0)-\theta_{1}(0)=u \epsilon_{1}, \\
\theta_{2}^{\prime}(0)-\theta_{1}^{\prime}(0)=-\epsilon_{1}\left(u^{2}+\omega\right), \\
-u\left(u^{2} \epsilon_{1}+\theta_{2}^{\prime}(0)\right)=Z \theta_{1}(0), \\
v_{1}(0)=v_{2}(0) \text { and } v_{1}^{\prime}(0)=v_{2}^{\prime}(0) .
\end{gathered}
$$




\subsection{General solution}

Introducing the linear differential operators:

$$
L_{1} \theta=\theta^{\prime \prime}+u \theta^{\prime}-\left(\omega+k^{2}\right) \theta, \quad L_{2} v=v^{\prime \prime}-k^{2} v,
$$

we re-write equations (5.65)-(5.68) as:

$$
\begin{gathered}
L_{1} \theta_{1}=0, \quad L_{2} v_{1}=-R_{p} k^{2} \theta_{1}, \\
L_{1} \theta_{2}=-u e^{-u z} v_{2}, \quad L_{2} v_{2}=-R_{p} k^{2} \theta_{2} .
\end{gathered}
$$

Since the perturbations decay at infinity, so that $v_{i}( \pm \infty)=0, \theta_{i}( \pm \infty)=0, i=1,2$, then the general solutions of (5.73)-(5.74) have the form:

$$
\begin{gathered}
v_{1}(z)=a_{1} \frac{R_{p} k^{2}}{k^{2}-\mu_{1}^{2}} \tilde{w}_{1}(z)+a_{2} \tilde{w}_{2}(z), \quad \theta_{1}(z)=a_{1} \tilde{w}_{1}(z), \\
v_{2}(z)=b_{1} w_{1}(z)+b_{2} w_{2}(z), \quad \theta_{2}(z)=b_{1} s_{1}(z)+b_{2} s_{2}(z),
\end{gathered}
$$

where $b_{1}, b_{2}, a_{1}, a_{2}$ are arbitrary constants and the functions $w_{i}, \tilde{w}_{i}, s_{i}$ for $i=1,2$ have the form

$$
\tilde{w}_{1}(z)=e^{\mu_{1} z}, \quad \tilde{w}_{2}(z)=e^{k z}, \quad w_{i}(z)=\sum_{j=1}^{\infty} a_{i, j} e^{\sigma_{i, j} z}, \quad s_{i}(z)=\sum_{j=1}^{\infty} c_{i, j} e^{\sigma_{i, j} z}
$$

with

$$
\begin{aligned}
& \mu_{1}=\frac{-u+\sqrt{u^{2}+4\left(\omega+k^{2}\right)}}{2}, \\
& \sigma_{1,1}=\frac{-u-\sqrt{u^{2}+4\left(\omega+k^{2}\right)}}{2}, \sigma_{2,1}=-k, \\
& \sigma_{i, j+1}=\sigma_{i, j}-u, \quad i=1,2, \quad j=1,2, \ldots \\
& c_{1,1}=1, \quad c_{2,1}=0, \quad a_{2,1}=1, \\
& a_{i, j}=\frac{R_{p} k^{2} c_{i, j}}{k^{2}-\sigma_{i, j}^{2}} \text { for }(i, j) \neq(2,1) \text { and } i=1,2, j=1,2, \ldots \\
& c_{i, j+1}=\frac{-u a_{i, j}}{\sigma_{i, j+1}^{2}+u \sigma_{i, j+1}-\left(\omega+k^{2}\right)} i=1,2, j=1,2, \ldots
\end{aligned}
$$

We assume here that

$$
k^{2}-\sigma_{i, j}^{2} \neq 0, \text { for } i=1,2, \quad j=1,2, \ldots, \text { and } k^{2}-\mu_{1}^{2} \neq 0,
$$

and

$$
\sigma_{i, j+1}^{2}+u \sigma_{i, j+1}-\left(\omega+k^{2}\right) \neq 0, \text { for } i=1,2, \quad j=1,2, \ldots
$$


It is easy to verify that the series (5.77) converge uniformly in $z$, and the solutions $\left(w_{i}, s_{i}\right)$, $i=1,2$ are linear independent if the numbers $\sigma_{i 1}$ are different.

We show now that the functions $\left(w_{i}, s_{i}\right)$ satisfy (5.73)-(5.74). Indeed, we denote

$$
w_{i}^{n}(z)=\sum_{j=1}^{n} a_{i, j} e^{\sigma_{i, j} z}, \quad s_{i}^{n}(z)=\sum_{j=1}^{n} c_{i, j} e^{\sigma_{i, j} z}, \quad i=1,2 .
$$

Substituting these partial sums into (5.73)-(5.74), we have

$$
L_{1} s_{i}^{n}=-u e^{-u z}\left(w_{i}^{n}-a_{i n} e^{\sigma_{i n} z}\right), \quad L_{2} w_{i}^{n}=-R_{p} k^{2} s_{i}^{n}, \quad i=1,2 .
$$

Passing to the limit as $n \rightarrow \infty$ we obtain that (5.77) satisfies (5.73)-(5.74).

\subsection{Dispersion relation}

The constants $a_{1}, a_{2}, b_{1}$ and $b_{2}$ should be found from the jump conditions (5.69)-(5.72). Simple computations lead us to the following linear system of equations

$$
\left\{\begin{array}{c}
b_{1} s_{1}(0)+b_{2} s_{2}(0)-a_{1}=u \epsilon_{1}, \\
b_{1} s_{1}^{\prime}(0)+b_{2} s_{2}^{\prime}(0)-a_{1} \mu_{1}=-\epsilon_{1}\left(u^{2}+\omega\right), \\
b_{1} s_{1}^{\prime}(0)+b_{2} s_{2}^{\prime}(0)+a_{1} \frac{Z}{u}=-u^{2} \epsilon_{1}, \\
b_{1} w_{1}(0)+b_{2} w_{2}(0)-a_{1} \frac{R_{p} k^{2}}{k^{2}-\mu_{1}^{2}}-a_{2}=0, \\
b_{1} w_{1}^{\prime}(0)+b_{2} w_{2}^{\prime}(0)-a_{1} \frac{R_{p} k^{2} \mu_{1}}{k^{2}-\mu_{1}^{2}}-a_{2} k=0 .
\end{array}\right.
$$

The solvability condition of this linear system allows us to obtain the dispersion relation. More precisely, from the second and the third equations we obtain

$$
a_{1}=\epsilon_{1} \frac{u \omega}{Z+u \mu_{1}} .
$$

From the first and the second equations

$$
\begin{aligned}
& b_{1}=\frac{\left(u \epsilon_{1}+a_{1}\right) s_{2}^{\prime}(0)-s_{2}(0)\left(a_{1} \mu_{1}-\epsilon_{1}\left(u^{2}+\omega\right)\right)}{s_{1}(0) s_{2}^{\prime}(0)-s_{2}(0) s_{1}^{\prime}(0)} \\
& b_{2}=\frac{s_{1}(0)\left(a_{1} \mu_{1}-\epsilon_{1}\left(u^{2}+\omega\right)\right)-s_{1}^{\prime}(0)\left(u \epsilon_{1}+a_{1}\right)}{s_{1}(0) s_{2}^{\prime}(0)-s_{2}(0) s_{1}^{\prime}(0)}
\end{aligned}
$$

and from the fourth equation

$$
a_{2}=b_{1} w_{1}(0)+b_{2} w_{2}(0)-a_{1} \frac{R_{p} k^{2}}{k^{2}-\mu_{1}^{2}} .
$$

Finally, the fifth equation provides the dispersion relation $\sigma\left(R_{p}, k, \omega, u, Z\right)=0$ as a compatibly relation which ensures that the system has a nonzero solution. 


\section{Stability conditions}

The dispersion relation allows the determination of eigenvalues of the linearized problem. If an eigenvalue $\omega$ lies on the imaginary axis and all other eigenvalues have negative real parts, then the dispersion relation determines the stability boundary. It is the cellular stability boundary if $\omega=0$, and the oscillatory stability boundary if $\omega=i \phi$ for $\phi \neq 0$. In the following subsections, we present the results corresponding to these two cases. The dispersion relation will be solved numerically. The series in (5.77) will be replaced by finite sums with a sufficiently large number of terms in such a way that the results would be independent of the truncation.

\subsection{Cellular stability boundary}

To find the cellular stability boundary, we put $\omega=0$ in system (5.23). We fix $u$ and find $R_{p}$ as a function of $k$ from the dispersion relation. We remark that the cellular instability boundary does not depend on $Z$. Indeed all coefficients of $Z$ in the dispersion relation vanish. Fig. 1 shows the critical Rayleigh number as function of the wavenumber $k$. The curve separates the stability and instability regions, that is the front is stable when $R_{p}<R_{p_{c r}}$ and unstable when $R_{p}$ is greater than its critical value. We note that for $R_{p}<0$, which corresponds to descending front [6], the front is always stable.

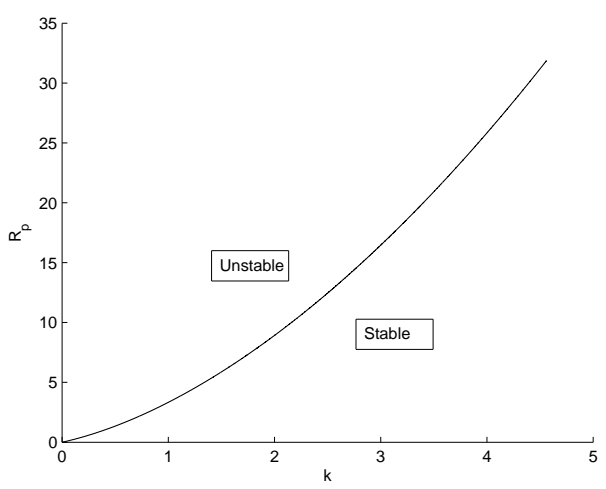

Fig. 1: Cellular stability boundary for $\mathrm{u}=\sqrt{2}$.

The eigenfunction corresponding to the zero eigenvalue at the stability boundary has the components that correspond to the temperature and to the stream function. The latter shows the convective pattern when the front loses its stability. Fig. 2 shows the level lines of the stream function for the values $R_{p}=30, u=\sqrt{2}, k=3.14$ and $k=1.57$, respectively (in the instability region). One or two vortex structure can emerge. 

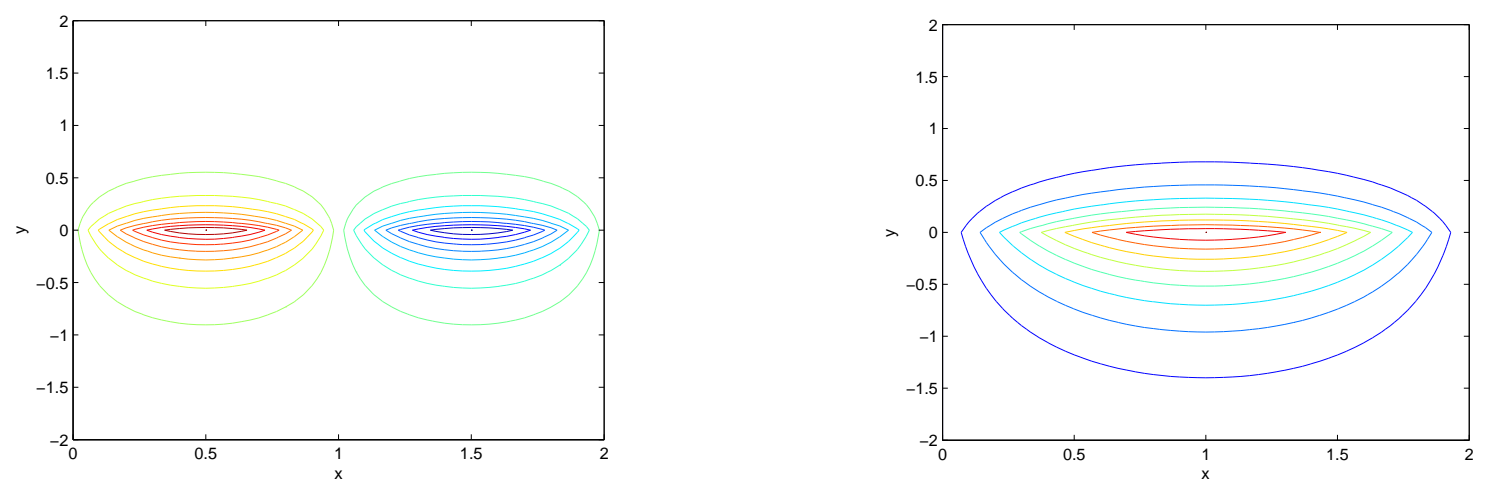

Fig. 2: Level lines for $R_{p}=30, k=3.14, u=\sqrt{2}$ (left) and for $R_{p}=30, k=1.57, u=\sqrt{2}$ (right).

When we increase the velocity $u$ of the stationary front, it becomes less stable (Fig. 3). There are two opposite phenomena related to the front speed. On the one hand, the temperature gradient near the front increases and promotes the convective instability $(T(x) \sim \exp (-u x))$. On the other hand, if the front speed increases, it makes more difficult for the perturbation to develop. It appears that the influence of the former on the front stability is more essential.

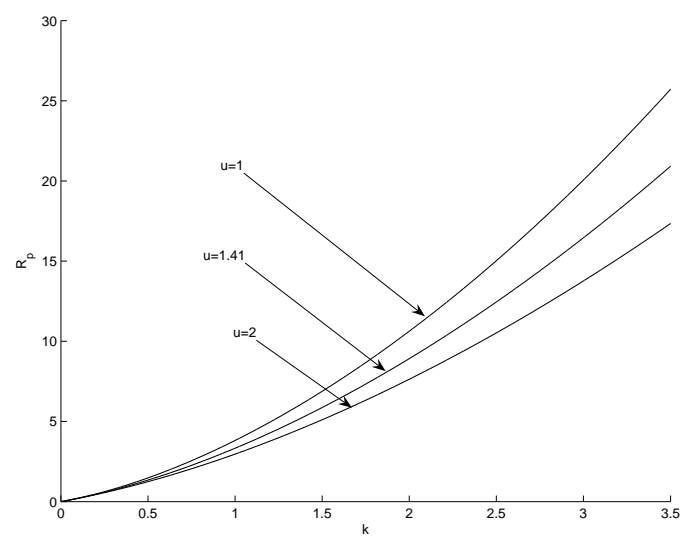

Fig. 3: Influence of $\mathrm{u}$ on the cellular stability boundary

\subsection{Oscillatory stability boundary}

To find the oscillatory stability boundary, we substitute $\omega=i \phi, \quad \phi \neq 0$ and $w_{i}, s_{i}$, $i=1,2$ given by (5.19) into (5.23). From the dispersion relation, we obtain a complex valued equation. Separating its real and imaginary parts, we obtain two real valued equations. We find from them the unknown frequency $\phi$ and the values of the parameters which give the stability boundary. We finally note that the dispersion relation is very complicated and should be analyzed numerically. 
Fig. 4 shows the critical values of the Zeldovich number as a function of the wavenumber for different values of the Rayleigh number. First, we can observe that when $k$ approaches zero the Zeldovich number tends to 8 , the same value as in the case without hydrodynamics $[6,7]$. It is known for condensed phase combustion [10] that the minimum of the neutral stability curve is reached for a positive value of $k$ (Fig $\left.4, R_{p}=0\right)$. This peculiarity of the problem results in appearance of spinning modes and other multi-dimensional regimes. It is interesting to note how convection influences the behavior of the stability boundary. For positive $R_{p}$ (upwards propagating front) and for sufficiently large $k$ the stability boundary moves down, that is the front is less stable. For smaller values of $k$ the behavior of the stability boundary becomes quite different. It has a sharp maximum for $k \approx 0.5$. For negative $R_{p}$ (downwards propagating front) the front becomes more stable for large $k$ and the minimum of the neutral stability curve moves to the left.

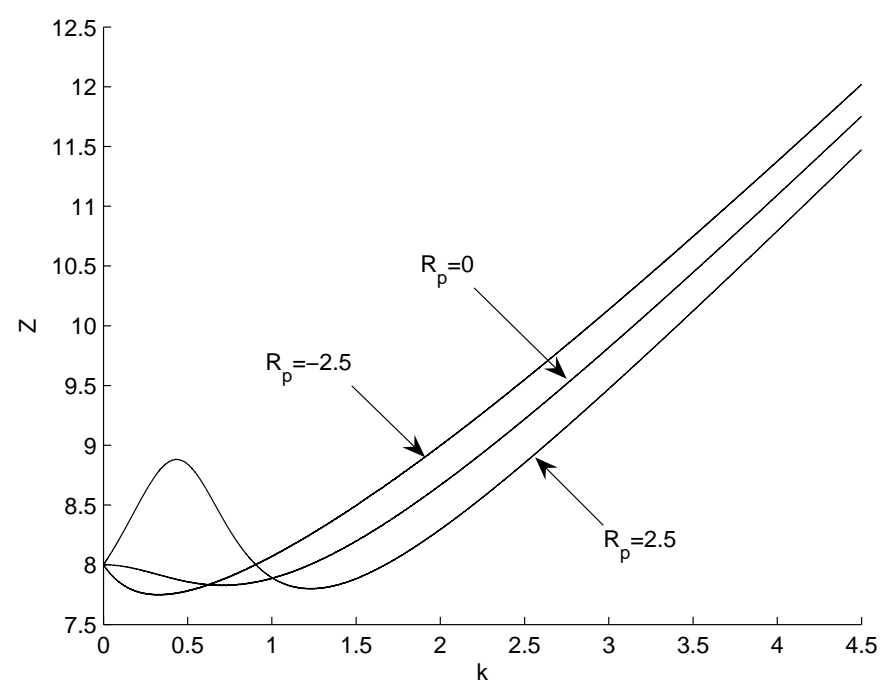

Fig. 4: Oscillatory stability boundary for $u=\sqrt{2}$

The structure of the vortices in the case of the oscillatory instability is shown in Fig. 5. It is different compared with the cellular instability. We recall that in this case the front moves upwards and at the same time there are hot spots that move along the front. This is the reason why the vortices also move along the front and have this specific form. The speed of their horizontal motion is determined by the frequency of oscillations $\phi$. For larger $\phi$ this speed increases and the vortices have more pronounced asymmetric form. For smaller $\phi$ they are almost symmetric. 

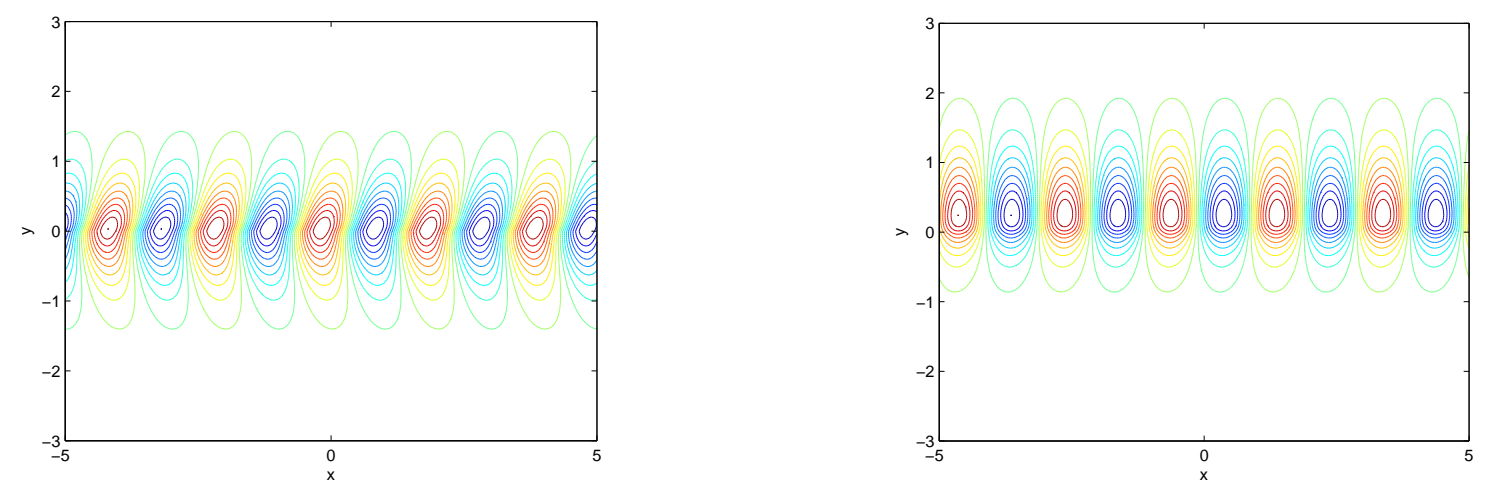

Fig. 5: Level lines when $k=3.14, u=\sqrt{2}$ for $R_{p}=10, Z=8.43, \phi=10.74$ (left) and for $R_{p}=17.66, Z=7.43, \phi=0.38$ (right).

The stability and the instability regions in the $\left(R_{p}, Z\right)$-plane are shown in Fig. 6 for the case of a bounded width which corresponds to the wavenumber $k=\pi$. The cellular stability boundary is given by the vertical line, it does not depend on the Zeldovich number. It corresponds to the zero eigenvalue of the linearized problem. The oscillatory instability boundary, where the corresponding eigenvalue is pure imaginary, decreases with the increase of the Rayleigh number $R_{p}$. When it approaches to the cellular instability boundary, the frequency of oscillations tends to zero (cf. Fig. 5, right).

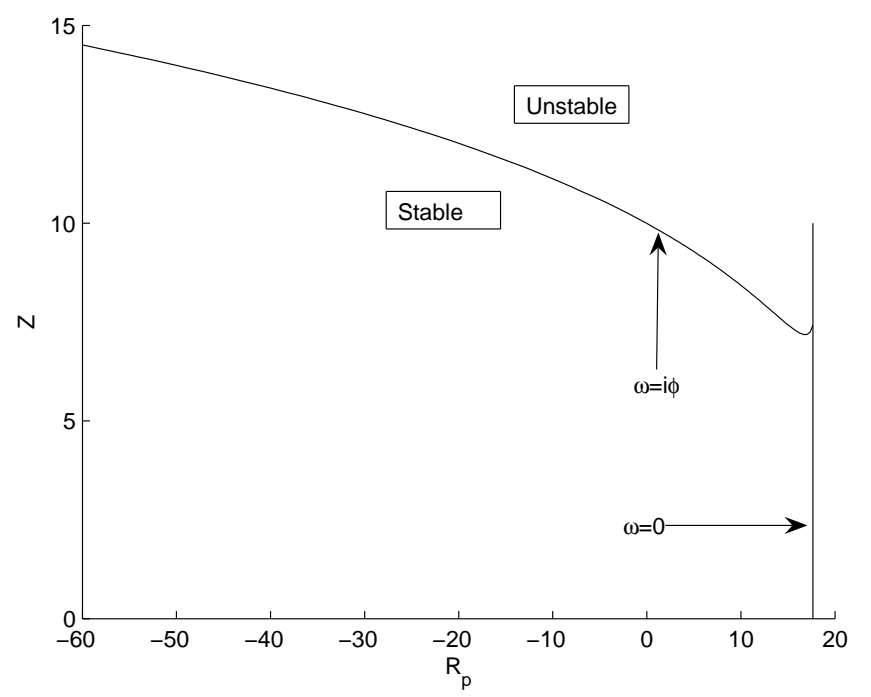

Fig. 6: Stability domain for $k=3.14$ and $u=\sqrt{2}$. 


\section{Direct numerical simulations}

This section is devoted to numerical simulations of problem (2.12)-(2.18) with the first order reaction. In the case of large activation energies $E, \delta$ in (2.16) is a small parameter. We replace it by zero. The model becomes

$$
\begin{gathered}
\frac{\partial \theta}{\partial t}+\mathbf{v} \cdot \nabla \theta=\Delta \theta+W_{Z}(\theta) \phi(\alpha) \\
\frac{\partial \alpha}{\partial t}+\mathbf{v} \cdot \nabla \alpha=\Lambda \Delta \alpha+W_{Z}(\theta) \phi(\alpha) \\
\mathbf{v}+\nabla p=R_{p}\left(\theta+\theta_{0}\right) \gamma \\
\nabla \cdot \mathbf{v}=0,
\end{gathered}
$$

with

$$
\begin{gathered}
W_{Z}(\theta)=Z^{2} e^{Z \theta}, \quad \phi(\alpha)=1-\alpha, \\
y \rightarrow+\infty, \quad \theta=-1, \quad \alpha=0 \text { and } \mathbf{v}=0, \\
y \rightarrow-\infty, \quad \theta=0, \quad \alpha=1 \text { and } \mathbf{v}=0
\end{gathered}
$$

This system of equations is studied numerically in a finite strip $\Omega=\left(0, L_{x}\right) \times\left(0, L_{y}\right)$ with homogeneous Neumann conditions at the boundary. The initial condition is chosen to be close to the one-dimensional traveling wave solution without convection. We will study the appearance of convective reaction fronts and will find the critical Rayleigh numbers.

Numerical computations are fulfilled using the stream function associated to the velocity. Due to incompressibility of the medium we can introduce the stream function $\psi$ defined by

$$
\mathbf{v}=\left(v_{x}, v_{y}\right)=\left(\frac{\partial \psi}{\partial y},-\frac{\partial \psi}{\partial x}\right)
$$

Function $\psi$ is then uniquely chosen by the condition

$$
\psi=0 \text { on } \Omega
$$

Therefore instead of system (7.1)-(7.4) we have system (7.1), (7.2) completed by the equation

$$
\Delta \psi=R_{p} \frac{\partial \theta}{\partial x}
$$

together with (7.6), boundary condition (7.7) and the homogeneous Neumann boundary condition for $\theta$ and $\alpha$.

We use a semi-implicit finite difference approximation and an alternating direction method. The basic set of parameters is $Z=8, \Lambda=0.1$ ( $\Lambda$ is the inverse of Lewis number), $L_{x}=2, L_{y}=35$. The initial condition is

$$
T(0, x, y)=T_{0}(y), \alpha(0, x, y)=\alpha_{0}(y), \psi(0, x, y)=0,
$$


where the functions $T_{0}$ and $\alpha_{0}$ are some step functions. We note that for small values of the Rayleigh number the convective regime develops slowly. As a consequence, the vortex can reach the top of the reactor without being completely developed. In this case the solution is shifted downwards and taken as a new initial condition. This procedure is repeated while the solution reaches a steady state regime.

We start the computations with a sufficiently large Rayleigh number with respect to its value at the stability boundary $\left(R_{p_{c r}}=26\right)$. Fig. 7 shows the level lines and the amplitude of the stream function (along a vertical section of the reactor) for $R_{p}=150$. For this value of the Rayleigh number, a convective regime rapidly appears and stabilizes as a travelling wave. We can also notice that the stream function is not symmetric with respect to the vertical middle line of the reactor. The appearance of a nonsymmetric regime can be related to a secondary bifurcation. For smaller values of the Rayleigh number it is symmetric (Fig. $8)$.
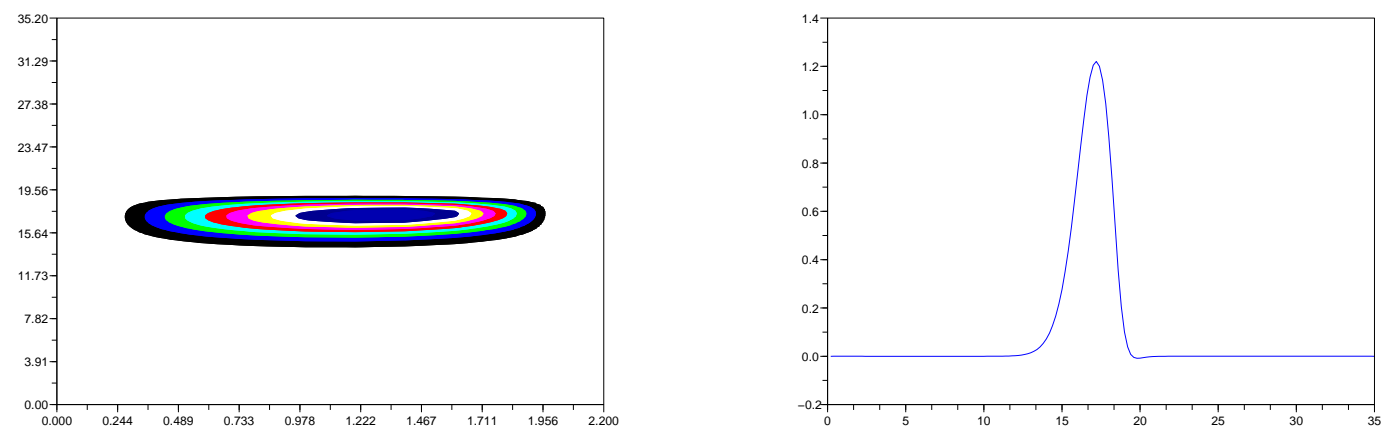

Fig. 7: Level lines (left) and amplitude (right) of the stream function for $R_{p}=150$.

Figures 8 and 9 show the stream function and the temperature for $R_{p}=30$ and for the stationary propagating front. It is interesting to note that because of the convection the temperature distribution is not symmetric with respect to the vertical middle line of the reactor and it is not monotone with respect to $y$.
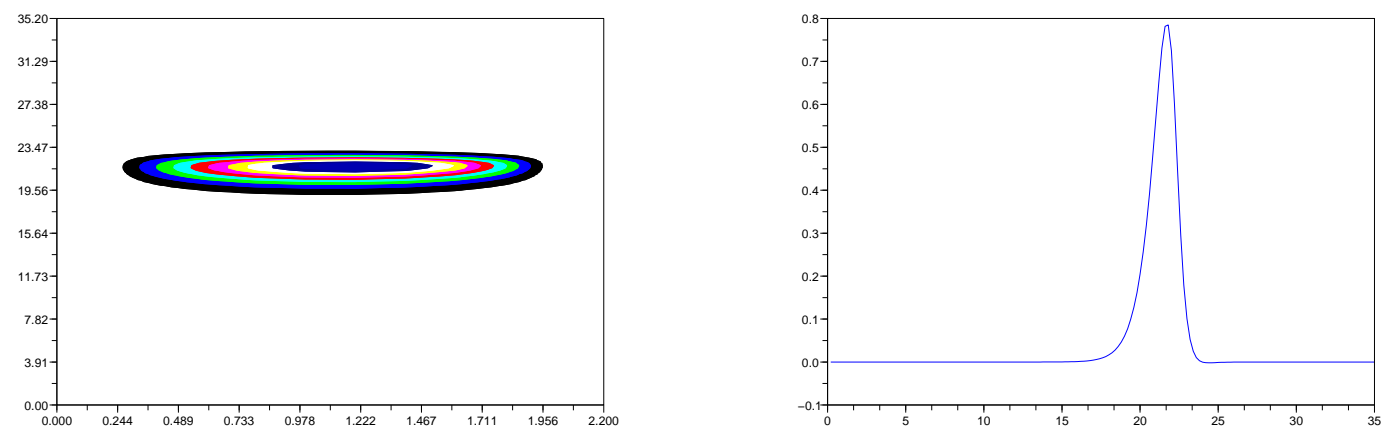

Fig. 8: Level lines (left) and amplitude (right) of the stream function for $R_{p}=30$. 

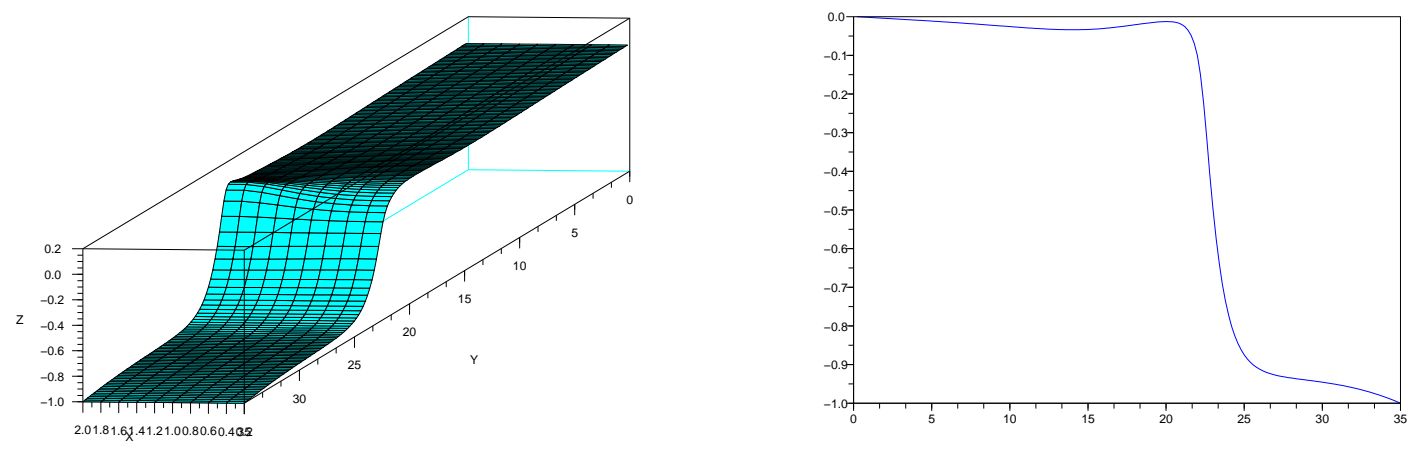

Fig. 9: Temperature distribution for $R_{p}=30$.

If we change the value $L_{x}=2$ to $L_{x}=4$ with the same values of other parameters, we obtain the critical value $R_{p_{c r}}=12$ of the Rayleigh number. The numerical simulations of the complete model are in agreement with the results of the linear stability analysis. In particular, $L_{x}=2$ (resp. $L_{x}=4$ ) corresponds to the wavenumber $k=\pi$ (resp. $k=\frac{\pi}{2}$ ) and the dispersion relation provides the critical value of the Rayleigh number about $R_{p_{c r}}=20$ (resp. $R_{p_{c r}}=8$ ). It is $R_{p_{c r}}=26$ (resp. $R_{p_{c r}}=12$ ) in the direct numerical simulations.

This correspondence is even better for larger values of the Zeldovich number. In fact, the critical value of the Rayleigh number found numerically converges to its value given by the linear stability analysis when $Z$ increases (see Table 1 ).

\begin{tabular}{|c|c|c|c|}
\hline$Z$ & 8 & 9 & 10 \\
\hline$R_{p_{c r}}$ & 26 & 22 & 20 \\
\hline
\end{tabular}

Table 1: Critical values of the Rayleigh number for various values of the Zeldovich number,

$$
L_{x}=2 \text {. }
$$

\section{Discussion}

In this work we study stability of reaction fronts in a porous medium. Natural convection can appear because of a nonhomogeneous temperature distribution and can result in a convective instability of the front. It can also influence the conditions of thermal instability.

For ascending fronts, because of the temperature gradient in front of the reaction zone, the exothermic chemical reaction can cause convective instability. It is similar to some extent to the classical Rayleigh-Benard problem of convection in a layer of a liquid heated from below. The condition of the cellular instability is independent of the Zeldovich number.

If the liquid is heated from above then it is known that the cellular instability does not occur. It is the same for downwards propagating fronts in a porous medium but it can be 
different for reaction fronts in a liquid phase where convective instability can occur even for descending fronts [6].

The results of the linear stability analysis show that the front becomes less stable when its velocity increases. It can be explained by the dependence of the temperature distribution on the velocity, $T(x) \sim \exp (-u x)$.

The oscillatory instability boundary corresponds to the case where a pair of complex conjugate eigenvalues with nonzero imaginary parts intersects the imaginary axis. The conditions of the thermal instability are influenced by convection. The front propagating upwards appears to be more stable with respect to multidimensional perturbations with small wavenumbers than in the pure combustion problem. The front propagating downwards appears to be less stable.

For perturbations with large wavenumbers, the situation is the opposite. The front propagating upwards appears to be less stable than in the problem without convection. The front propagating downwards is more stable. The influence of convection on the thermal instability for reaction fronts in a porous medium is similar to that for reaction fronts in a liquid phase [6].

\section{References}

[1] Aldushin A.P. and Merzhanov A.G. Filtration combustion of metals. In Matros Yu. Sh. (Ed.), Propagation of thermal waves in heterogeneous media. Nauka, Novosibirsk, 1988, 9-52.

[2] Aldushin A.P. Theory of filtration. In Matros Yu. Sh. (Ed.), Propagation of thermal waves in heterogeneous media. Nauka, Novosibirsk, 1988, 52-71.

[3] Brailovsky I. and Sivashinsky G. On progation limits in porous media combustion, Combust. Theor. Modelling 6, (2002) 595-605.

[4] De Wit A. Fingering of chemical fronts in porous media, Phys. Rev. Lett. 87, (2001) 054502.

[5] De Wit A., Kalliadasis S. and Yang J. Fingering instabilities of exothermic reactiondiffusion fronts in porous media, Physics of fluids vol. 16 n. 5, (2004) 1395-1409.

[6] Garbey M., Taik A. and Volpert V. Influence of natural convection on stability of reaction fronts in liquids, Quart. Appl. Math. 53, (1998) 1-35.

[7] Garbey M., Taik A. and Volpert V. Linear stability analysis of reaction fronts in liquids. Quart. Appl. Math. 54 (1996), No. 2, 225-247.

[8] Landau L.D. The theory of slow combustion. J.Exper. Theor. Physics, (1944), v.14, p. 240 
[9] Landau L.D. and Lifshitz E.M. Fluid Mechanics, Pergamon, Oxford, 1987.

[10] Makhviladze G.M. and Novozhilov B.V. The two-dimensional stability in condensed phase combustion. Prikl. Mech. Techn. Fiz., (1971), No. 5, 51-59 (Russian). English translation in J. Appl. Mech. Tech. Physics.

[11] Matkowsky B.J. On flames as discontinuity surfaces in gasdynamic flows. A celebration of mathematical modeling. 137-160, Kluwer Acad. Publ., Dordrecht, 2004.

[12] O’Malley R.E. Introduction to the simgular perturbations, Appl. Math. and Mech. Volume 41, Academic Press, Inc. New York. London.

[13] Nayfeh A.H. Perturbation Methods, Wiley, New York 1973.

[14] Merzhanov A.G. Solid flames: Discoveries, concept and horizons of cognition, Combust. Sci. and Tech. 98, (1994) 307-336.

[15] Volpert A., Volpert Vit. and Volpert Vl. Travelling wave solutions of parabolic systems. AMS, Providence, 1994.

[16] Zeldovich Ya.B., Barenblatt G.I., Librovich V.B. and Makhviladze G.M. The mathematical theory of combustion and explosions. Plenum, New York, 1985. 